\title{
Enterprise Financial Leverage and Risk Assessment Based on Mobile Payment under Artificial Intelligence
}

\author{
Xiaoling $\mathrm{Xu}^{1}$ and Jianghao Song $\mathbb{D}^{2}$ \\ ${ }^{1}$ Guangdong Nanhua Vocational College of Industry and Commerce, Guangzhou, Guangdong, China \\ ${ }^{2}$ Guangdong Vocational Institute of Sport, Guangzhou, Guangdong, China \\ Correspondence should be addressed to Jianghao Song; songjh@nhic.edu.cn
}

Received 8 October 2021; Accepted 8 November 2021; Published 9 December 2021

Academic Editor: Sang-Bing Tsai

Copyright (C) 2021 Xiaoling Xu and Jianghao Song. This is an open access article distributed under the Creative Commons Attribution License, which permits unrestricted use, distribution, and reproduction in any medium, provided the original work is properly cited.

\begin{abstract}
To better promote the healthy and long-term development of corporate financial management, the basement is established on the perspective of artificial intelligence (AI). Initially, based on the theories of modern mobile payment (MP) and corporate financial leverage, the corresponding data set is obtained through the questionnaire method as the research data. The reliability coefficients obtained after the test are all above 0.65 , indicating that the reliability and stability of the entire data are relatively good. Besides, it is also found from the data of the questionnaire that some residents believe that MP will bring harm such as information leakage. Next, a new multilevel evaluation analysis method is introduced. After evaluating the financial management risk, operation risk, and network security risk existing in enterprise MP, it is found that the financial management risk accounts for the largest proportion of the three, with a risk weight of about 0.54 , and the capital risk occupies the main position in the financial management risk. Finally, through the analysis of the risks existing in the whole operation process of the enterprise, it is found that about $50 \%$ of the financial management risk of the enterprise in the market belongs to the advanced risk, about 30\% of the operational operation risk belongs to the low risk, and about $20 \%$ of the network security risk belongs to the advanced risk, which indicates that the financial management risk and network security risk are the top priority of the enterprise MP risk. Although the operational operation risk belongs to the low risk, it cannot be ignored. Subsequently, feasible suggestions and opinions are put forward on these phenomena from the perspectives of the government, enterprises, and residents. Therefore, there is great reference significance for the current financial risk assessment of enterprises based on MP.
\end{abstract}

\section{Introduction}

At present, the continuous development of Internet technology has given birth to the era of artificial intelligence (AI). In turn, the vigorous development of AI has provided a good opportunity for mobile payment (MP). The development of MP has replaced the traditional ways of payments in clothing, food, housing, and travel. In daily life, there are platforms such as Taobao and T-mall for shopping, Meituan and Elema for eating, and Didi and Cao Cao for traveling. MP continues to create convenience for human beings [1]. However, MP is also constantly bringing risks to the development of social market. Dan (2018) found that the payment and sales environment of MP was unsafe through the investigation and analysis of consumers' satisfaction with
MP [2]. Pi (2018) pointed out that the cash-out problem at present was quite serious. Relevant laws and regulations should be improved to provide a healthy payment environment for the market [3]. Zhou (2018), taking Alipay as an example, through dealing with the accounting business problems of MP, suggested that enterprises should increase the setting of accounting secondary items to reduce the hidden dangers caused by capital risks [4]. Kahn (2019) talked about the connection between the economics of network security of MP and other fields, clarified the security consideration of MP, and put forward that legislative supervision was the focus of work [5]. Globally, the risks of MP are involved, and all of them mention strict supervision to create a healthy payment environment [6-8]. Jiang (2018) believed that there were two main types of risks in third- 
party mobile payment at this stage: one is the risk of the industry itself, including the risk of funds in transit, criminal risk, and information disclosure risk; the other is the industry stage risk, which is related to the development characteristics of the industry, including single fault risk and financial business risk. Single failure risk refers to the risk that industry monopolists have problems that have a huge impact on the entire market. Financial business risk refers to the risk formed by the platform's cooperation with other financial institutions in asset allocation [9]. The research of Thomas (2019) on quantum algorithms enabled quantum computers to implement the Shor algorithm in a scalable way for the first time in history. Scalable quantum systems derived from new research can break down the mass factor extremely efficiently. This means that the traditional RivestShamir-Adleman (RSA) encryption method based on the large integer factorization problem is no longer safe in theory, and the risk of being decoded is greatly increased [10]. Cao (2020) analyzed the risks of WeChat payment and concluded that the risks of WeChat payment mainly include personal information risk, payment method risk, and confidentiality technology risk [11]. Hao (2019) analyzed the legal risks of third-party mobile payment from the perspectives of user information and privacy security, account fund security, and financial legal risks. User information and privacy security refer to the risk of theft of user information and privacy caused by imperfect technology and system vulnerabilities of third-party mobile payment platform. Account fund security risk refers to the risk of property damage caused by hackers' invasion or users' mismanagement of accounts. Financial legal risk refers to the legal risk of precipitation funds and money laundering of the platform. In terms of technical risks, mobile payment can be implemented in three ways, one based on Store Management Service (SMS), one based on With Particular Average (WPA), and the other based on Near Field Communication (NFC). At present, the most popular is NFC technology. NFC technology is a short-range wireless communication technology, which can realize two-way communication between the two devices. Due to the high security of NFC technology, NFC technology can be widely used in the mobile payment industry [12]. Chen (2019) divided the technical system into three aspects: application layer, transaction layer, and payment processing layer and then analyzed the possible payment security risks of mobile payment from three aspects: terminal, wireless network, and payment process [13]. Guo (2018) put forward two suggestions to resolve the risks of third-party mobile payment from the perspective of establishing a legal guarantee system. First, relevant departments should learn from the legislative experience of foreign electronic payment and formulate a mobile payment basic law that meets the actual situation in China as soon as possible. Second, the judicial proceedings should also be appropriately reformed. The above experts and scholars have analyzed the risks of mobile payment and corporate financial leverage from different directions. These theories have a profound impact on corporate financial management. Here, these methods are optimized while drawing lessons from them. And a new multilevel evaluation method is proposed, which not only accurately analyzes the proportion of financial management risks, operational risks, and network security risks in financial risk assessment but also provides new ideas for future research.

Under such a background, starting from the background of AI era, analysis is made on the data obtained from the questionnaire by using multilevel evaluation analysis. Through the study of evaluation of financial management risk, operational risk, and network security risk, it is known that the MP risk in the market is serious, and it is the common demand for everyone to create a healthy and secure mobile environment.

\section{Materials and Methods}

\subsection{Related Theoretical Basis}

2.1.1. MP. MP refers to the process that consumers use mobile phones, bracelets, and other electronic products in their daily life to complete payment of the commodity [14]. With the advent of AI, the application scenarios of MP are constantly expanding [15]. MP is a brand new payment method formed by the combination of four channels: Internet, mobile client, terminal equipment, and financial institutions [16]. From the definition, it can be found that MP has the following characteristics: (1) convenience: compared with traditional consumption methods, MP does not need to consider the trouble of changing change. It also can accurately realize a series of services such as inquiry, payment, transfer, and recharge; (2) mobility: MP can complete various functions at any time and place, and it is no longer necessary to consider the commuting time between relevant financial institutions, or pay face-to-face between consumers and sellers; and (3) high comprehensive degree: MP can be used not only for monetary payment but also for payment of water, electricity, gas, and Internet charges. Besides, it can also be used for online shopping and so on [17-19].

The era of AI has brought infinite vitality to the development of MP, which in turn enriches the types of $\mathrm{AI}$ technology [20, 21]. At present, consumers, business men, and government policies are encouraging the development of MP. According to the 2020 MP Security Survey Report released by China UnionPay in February 2021. In 2020, each person in China used MP three times a day on average, which shows that MP has a very large user group, which can be divided into the following four types according to the different operation modes [22, 23].

(1) Operator-oriented mode, in which financial institutions are no longer required to participate, and consumers must complete various transactions in accounts specially set by operators for them [24]. Figure 1 signifies the operator-oriented mode.

(2) The third-party payment institution is the main mode, which is independent from financial institutions. The third party can make consumers complete various payments by entering this interface through its own technical operation, economic prejudgment, 


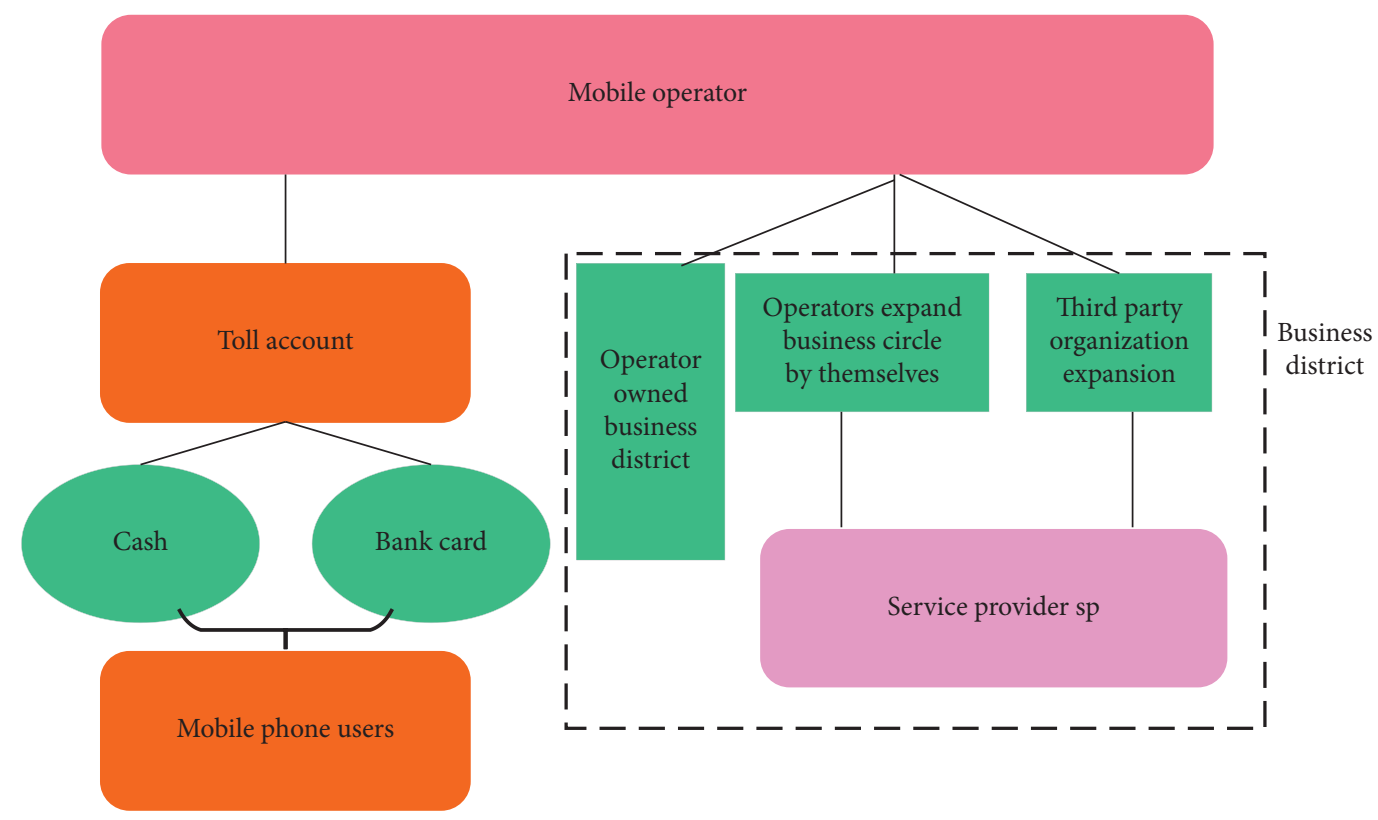

Figure 1: The operator-oriented MP mode.

and capital operation $[25,26]$. Figure 2 displays the third-party payment mode.

(3) The mode based on financial institutions, which mainly completes various services through mobile banking, requires the combination of financial institutions, consumers, terminal equipment, and communication operations [27]. Figure 3 explains MP mode based on financial institutions.

2.1.2. Enterprise Financial Leverage. Enterprise financial leverage refers to the phenomenon that the existence of fixed debt interest and preferred stock dividend significantly impacts the sovereign capital of common stock. Fixed debt interest and preferred stock dividends are leverage, while common stock sovereign funds are that pivot point [28]. The financial risk can be reflected from the degree of financial leverage of enterprises. The greater the coefficient of financial leverage, the greater the financial risk and vice versa [29].

\subsection{Research Method}

2.2.1. Data Collection Method. (1) Data collection method: due to the writing needs, first, China Knowledge Network, Google Academic, and other information channels are used for data query. Second, there are many articles and works from columnists and related Internet information, which provide theoretical basis [30, 31]. (2) Qualitative and quantitative analysis: by collecting basic theoretical knowledge related to MP, a collection is made of the factors affecting the financial leverage risk of enterprises. A questionnaire is designed to obtain relevant data, and the results are analyzed [32-35]. (3) Questionnaire survey method: by setting up corresponding questionnaire questions to understand the factors affecting the financial leverage risk of enterprises, 310 questionnaires are randomly distributed to the public and enterprises, respectively, in June 2019 and August 2020. In order to be ensured of the scientific essential of the questionnaire, the questionnaire is especially discussed together with experts from related specialties before the questionnaire is distributed and modified with the unreasonable contents in the questionnaire. In order to ensure the corresponding recovery rate, the form of distribution in person is taken and questionnaire is recovered on the spot, with a recovery rate about $90.3 \%$, an effective recovery of 252 pieces, and an effective recovery rate of $81.3 \%$. Figure 4 shows the specific investigating steps.

In order to make the results of the questionnaire more accurate, the validity test of systematic error variance is introduced as Equation (1).

$$
r=\frac{\sum X Y-\left(\sum X \sum Y / N\right)}{\sqrt{\sum X^{2}-\left(\left(\sum X\right)^{2} / N\right) \sqrt{\sum Y^{2}-\left(\left(\sum X\right)^{2} / N\right)}}} .
$$

In Equation (1), $r$ means reliability coefficient, $X$ refers to a dependent variable, $Y$ is an independent variable, and $N$ indicates the quantity.

Equation (1) displays the fractional difference of actual numbers, as given in Equation (2).

$$
\alpha=\frac{K}{K-1}\left(1-\frac{\sum_{i=1}^{K} \sigma_{Y i}^{2}}{\sigma_{X}^{2}}\right) .
$$

In Equation (2), $\alpha$ is coefficient, $K$ indicates quantity, $i$ indicates the $i_{\text {th }}$ real number, $\sigma$ represents a random variable, and the meaning of other letters is the same as in Equation (1). Generally speaking, the higher the reliability coefficient, the higher the reliability between variables, namely, the higher the internal consistency between variables. When $\alpha$ is less than or equal to 0.3 , it indicates that the variable is not credible. When it is between 0.3 and 0.4 , the variables are 


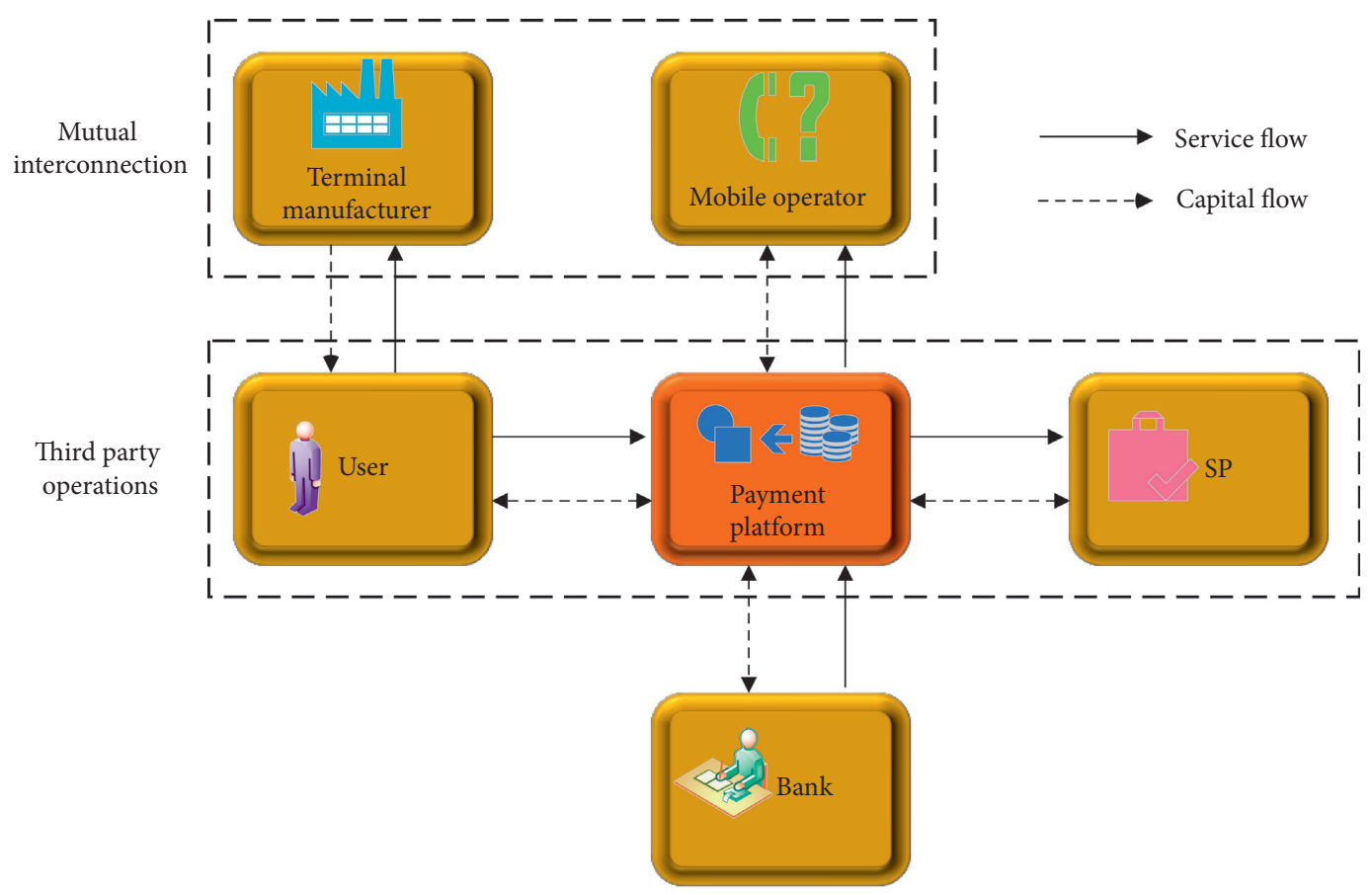

FIGURE 2: MP mode based on third-party payment institutions.

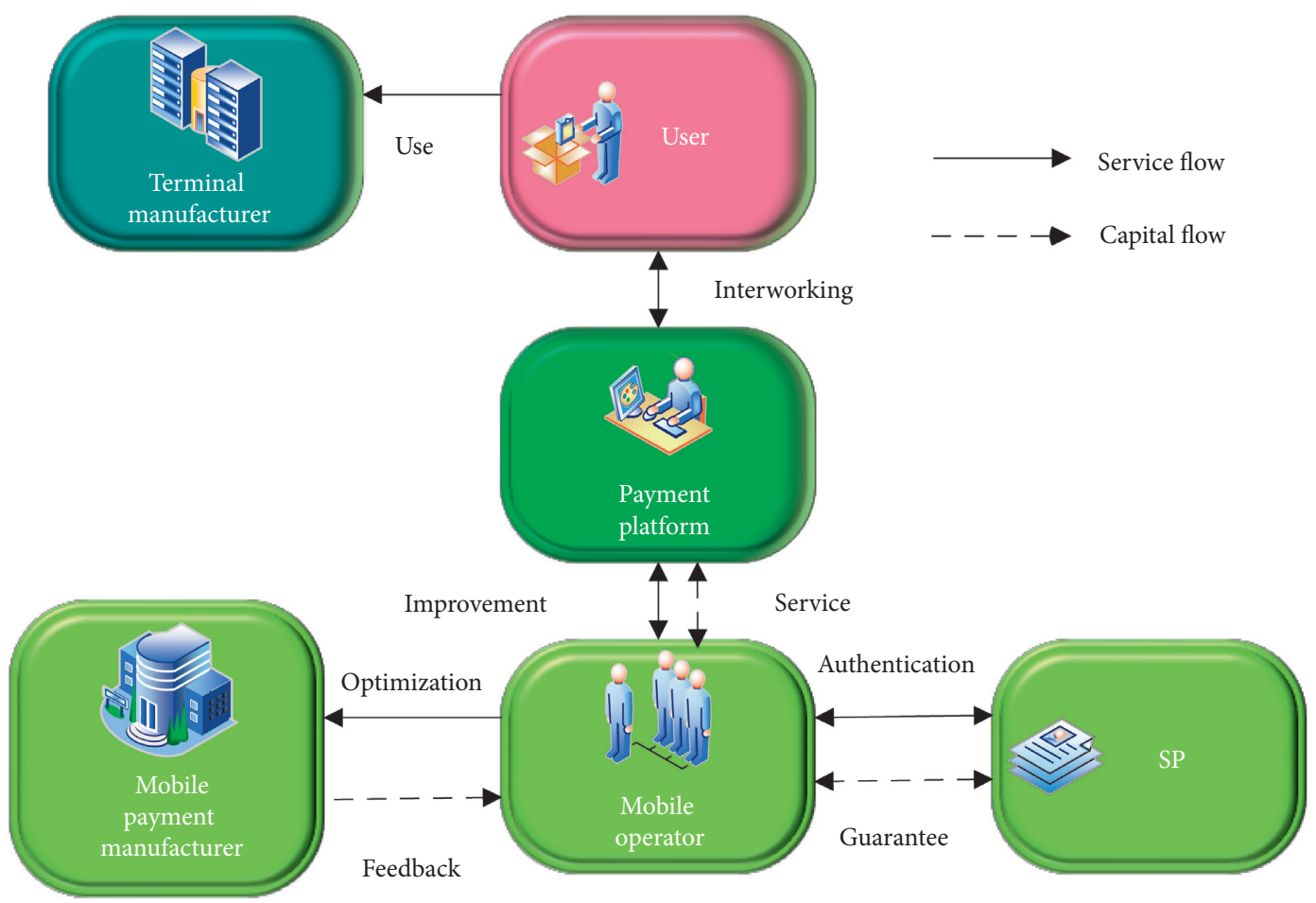

Figure 3: MP mode based on financial institutions.

initially credible. When it is between 0.4 and 0.5 , there is a slight confidence between variables. When $\alpha$ verifies between 0.5 and 0.7 , the variables are reliable. When $\alpha$ changes between 0.7 and 0.9 , the variables are more reliable. And when $\alpha$ is more than 0.9 , the variables are most reliable.
2.2.2. Sample Determination. On the basis of the above questionnaire, the risks of MP in enterprises are divided into four categories: financial management risks, operational risks, market security risks, and network security risks. Table 1 displays the details. 


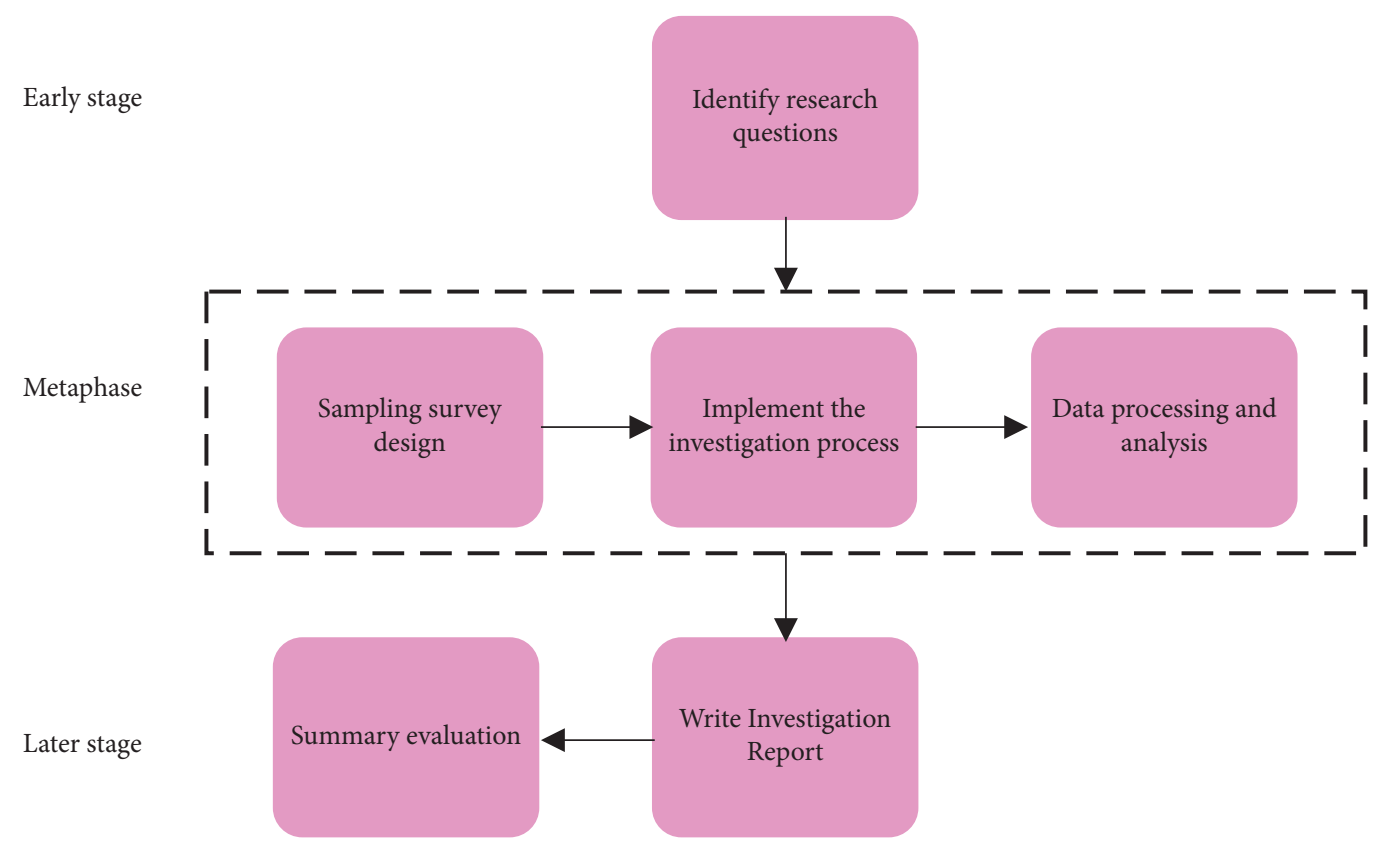

FIGURE 4: Specific investigating steps.

TABLE 1: Index evaluation system of MP risk.

\begin{tabular}{lc}
\hline Primary type & Secondary type \\
\hline Financial management risk A1 & $\begin{array}{c}\text { Capital risk A11 } \\
\text { Money laundering risk A12 } \\
\text { Cash-out risk A13 }\end{array}$ \\
\hline Operational risk A2 & Platform operation risk A21 \\
& Credit risk A22 \\
& Reputational risk A23 \\
\hline Network security risk A3 & Information security risk A31 \\
& Software and hardware risk A32 \\
& Business innovation risk A33 \\
\hline
\end{tabular}

According to Table 1, matrix A can be obtained as Matrix (3).

$$
A=\left[\begin{array}{cccc}
1 & 2 & 4 & (1 / 2) \\
(1 / 2) & 1 & 2 & 1 \\
(1 / 4) & (1 / 2) & 1 & (1 / 2) \\
2 & 1 & 2 & 1
\end{array}\right]
$$

By using the same method, the first-level matrices A1, A2, and A3 can be obtained, as Matrix (4), Matrix (5), Matrix (6), and Matrix (7), respectively.

$$
\begin{aligned}
A 1 & =\left[\begin{array}{cccc}
1 & 1 & 5 & 3 \\
1 & 1 & 5 & 3 \\
(1 / 5) & (1 / 3) & 1 & (1 / 3) \\
(1 / 3) & (1 / 3) & 3 & 1
\end{array}\right], \\
A 2 & =\left[\begin{array}{ccc}
1 & 5 & 2 \\
(1 / 5) & 1 & (1 / 3) \\
(1 / 2) & 3 & 1
\end{array}\right],
\end{aligned}
$$

$$
\begin{aligned}
& A 3=\left[\begin{array}{cccc}
1 & 3 & 2 & 6 \\
(1 / 3) & 1 & 1 & 3 \\
(1 / 2) & 1 & 1 & 3 \\
(1 / 6) & (1 / 3) & (1 / 3) & 1
\end{array}\right], \\
& A 4=\left[\begin{array}{ccc}
1 & (1 / 3) & 3 \\
3 & 1 & 5 \\
(1 / 3) & (1 / 5) & 1
\end{array}\right] .
\end{aligned}
$$

\section{Results}

3.1. Development Status of MP. According to relevant research statistics, in 2010, there were more than 700 million mobile phone users in China, and 250 million people opened the mobile Internet access function, which were the "main force" of MP. Figure 5 displays the transaction scale of China MP from 2013 to 2019.

Figure 5 indicates that the scale of China's MP transactions has been expanding continuously from 2013 to 2019, and MP has developed rapidly in just seven years, especially in 2016, when the growth rate of transaction scale reached $379 \%$. In 2019, when the transaction scale reached 281.2 trillion yuan, there were still many potential users of MP in China. And in the future, with the continuous upgrading of AI technology, there will be more and more MP users.

Due to the difference in distance, MP can be divided into remote payment and near-field payment. Figure 6 indicates the comparison of transaction volume between them from 2015 to 2019.

At present, with the continuous integration of online trading platforms such as Taobao and JD.COM into people's production and life, remote payment is mainly used. This has a great relationship with the characteristics of $\mathrm{MP}$, which will not be affected by time, place, weather, season, and other 


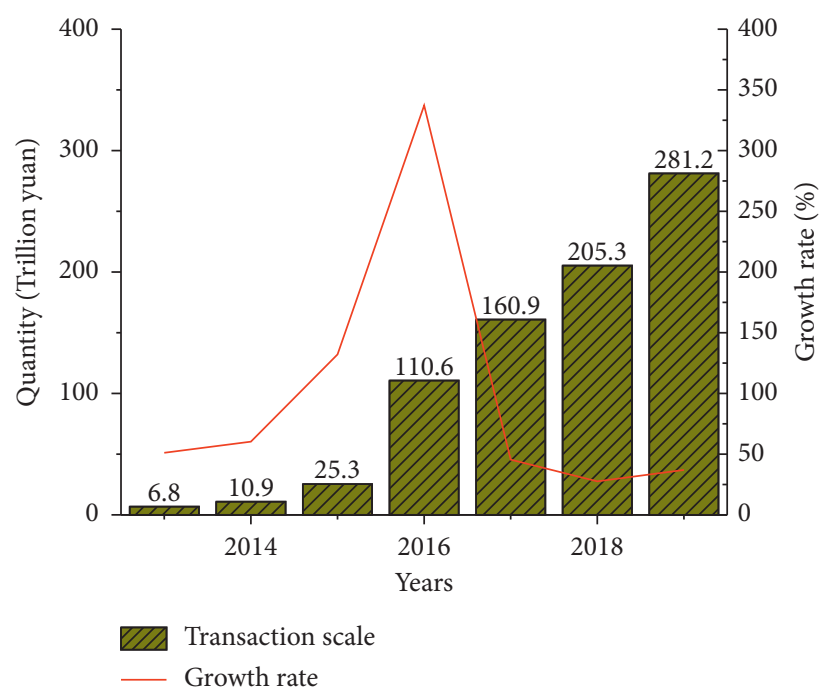

Figure 5: China MP transaction scale from 2013 to 2019.

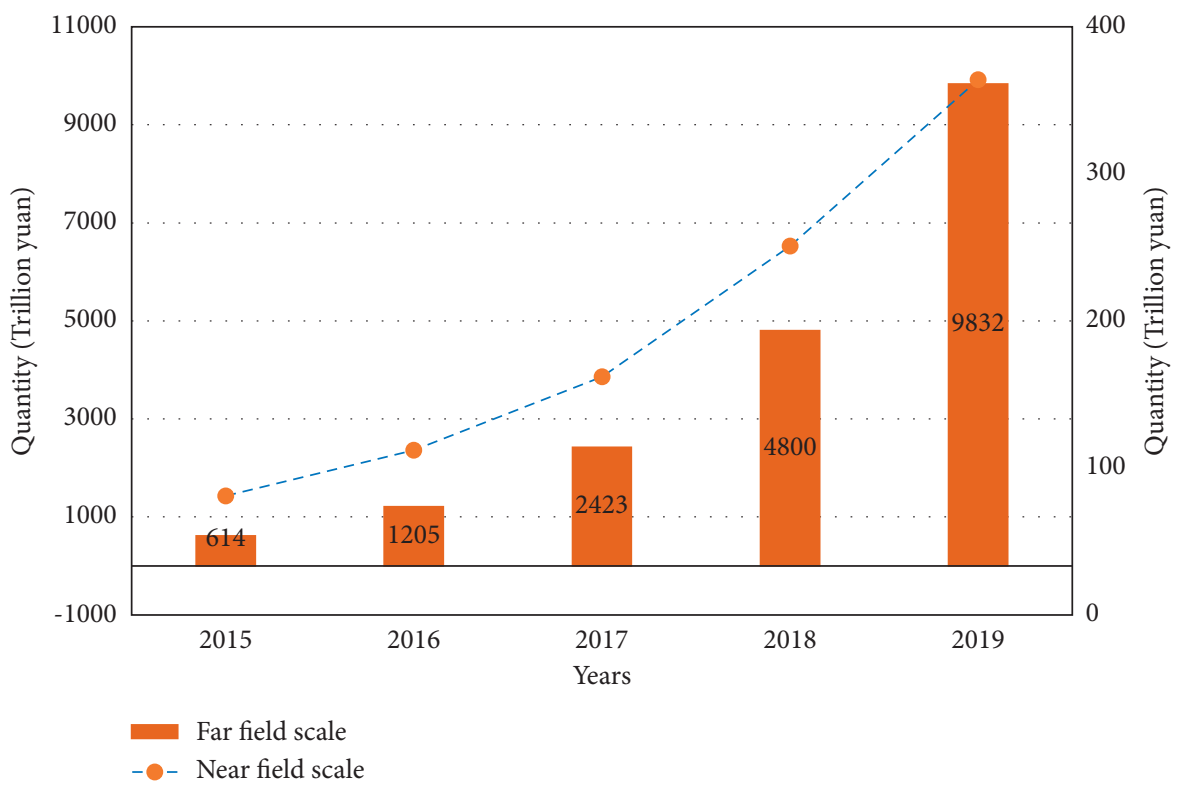

Figure 6: Scale of China MP of remote and near-field transactions from 2015 to 2019.

factors. In the past five years, the transaction volume of nearfield payment has not been high, which is closely related to the current business model and the global technical level.

3.2. Risk Assessment of Enterprise Financial Leverage under $M P$. First of all, the questionnaire method is briefly introduced, and the results of these questionnaires are analyzed. Table 2 shows the statistical information of samples.

A total of 252 questionnaires were collected this time. Table 2 indicates that in terms of gender, there are 152 female respondents and 100 male respondents. In terms of age, about $78 \%$ of the total number of people are aged $20-35$ years, followed by students aged 0-20 years, accounting for about $12.7 \%$. People aged 35-45 years and over 45 account for almost $3.6 \%$ to $4 \%$, respectively. Young people account for the most. About 51.6\% respondents have used MP, which is slightly more than the number of people who have never used. This shows that half of the people still believe in the popular MP.

The standard deviation can reflect the distribution range of the measured score. The larger the standard deviation is, the problem item can identify the more differences reflected by individuals. Figure 7 displays the results of standard deviation of sample data of the above questionnaire.

In Figure 7, F1 represents the fear of paying more money to others due to one's own misoperation in the process of using MP; F2 indicates the fear of revealing the financial information of one's own enterprise or individual in the process of using MP; F3 stands for that one's own money will be paid but the purchased items will not be received; and F4 means that if the mobile equipment used is stolen or lost, it 
TABLe 2: Distribution table of sample statistical information.

\begin{tabular}{|c|c|c|c|}
\hline Features & Type & Number of people & Proportion (\%) \\
\hline \multirow{2}{*}{ Gender } & Female & 152 & 60.4 \\
\hline & Male & 100 & 39.6 \\
\hline \multirow{4}{*}{ Age } & $0-20$ years old & 32 & 12.7 \\
\hline & $20-35$ years old & 201 & 80 \\
\hline & $35-45$ years old & 10 & 4 \\
\hline & Over 45 & 9 & 3.3 \\
\hline \multirow{2}{*}{ MP usage history } & Used & 130 & 51.6 \\
\hline & Not used & 123 & 48.4 \\
\hline \multirow{2}{*}{ Individual or enterprise } & Personal & 162 & 64.3 \\
\hline & Enterprise & 90 & 35.7 \\
\hline
\end{tabular}

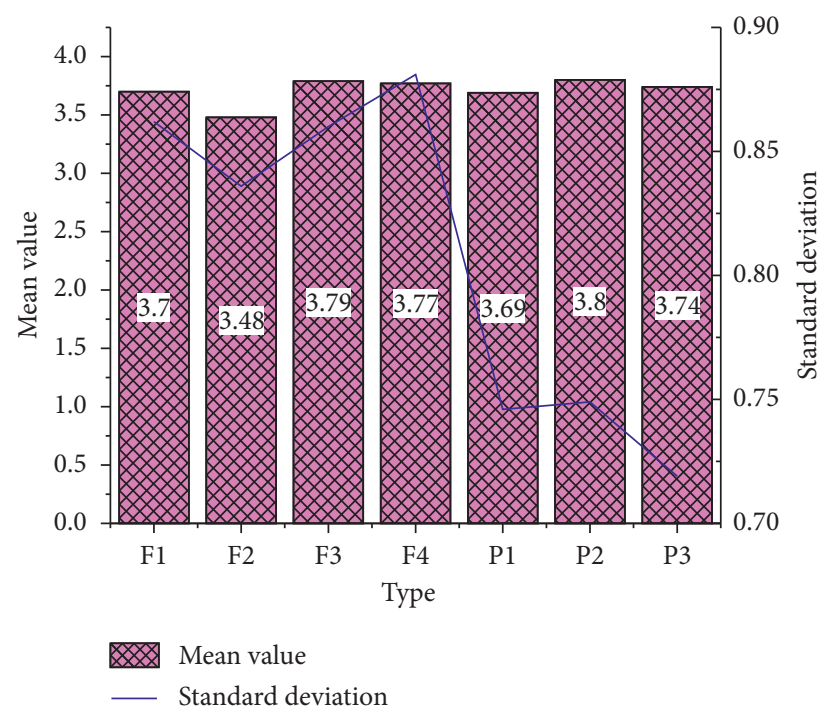

Figure 7: Mean and standard deviation of questionnaire data.

will cause unnecessary losses to users' property. P1 indicates that when an enterprise or individual chooses MP, his financial information is intercepted. P2 indicates that in the process of choosing MP, personal information is shared or traded without the user's consent. P3 represents that when enterprises or individuals choose MP, their consumption information is recorded, and their privacy rights are violated. As Figure 7 shows, among the financial risks F1-F4, individuals and enterprises are most concerned about the property losses caused when their MP devices are stolen or lost causing that both property and money are gone. The next factor is the misoperation of individuals or enterprises to pay more money. And the last factor is the disclosure of information. Compared with F1-F4, the standard deviation in $\mathrm{P} 1-\mathrm{P} 3$ is much reduced, which means that both enterprises and individuals have their own financial risks, especially when their property is stolen.

According to the reliability analysis equation introduced above, the reliability analysis results of F1-F4 and P1-P3 can be obtained, as shown in Figure 8.

Figure 8 indicates that there is correlation among the data variables in the questionnaire, and the reliability coefficient of P1-P3 is obviously higher than that of F1-F4, which shows that there is a high degree of internal

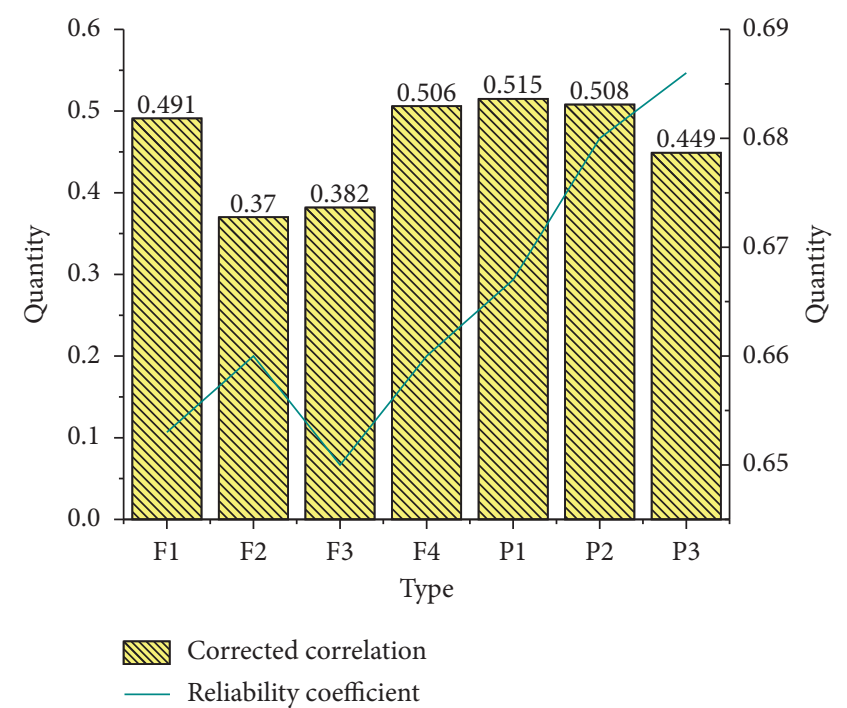

FIgURE 8: Reliability analysis of questionnaire data.

consistency among the variables in P1-P3. Among them, the average reliability coefficient is about 0.656 in financial risks and about 0.677 in privacy risks, which means that the reliability and stability of the whole data are relatively good.

According to the results of the above questionnaire, the risk weights of the primary type and the secondary type are scored, which are "highest," "higher," "medium," "lower," and "low." Figure 9 displays the specific results.

According to the MP risk evaluation index system described in Table 1 and the classification of risk weights, it can be clearly observed from Figure 9 that the highest risk weight lies in A11, followed by A12, A13, A21, A32, A23, A31, A22, and finally A33. This shows that among the financial management risk, operational operation risk, and network security risk, the weight of financial management risk is the highest, and the weight of operational operation risk and network security risk is much different. Hence, financial management risk is particularly important in risk assessment. Additionally, capital risk and money laundering risk account for the main position in financial management risk, and then cash risk. This shows that these risks must be prevented in advance; otherwise, the difficulty of financial management will gradually increase. 

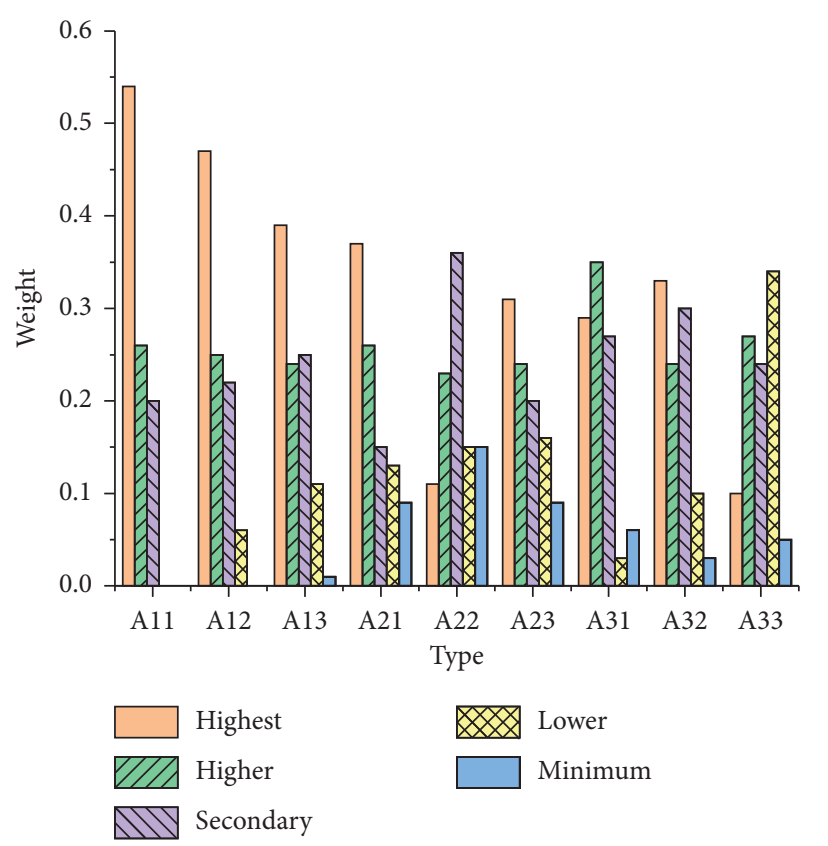

FIGURE 9: Risk weights results of questionnaire.

Figure 9 shows that among financial management risks, operational risks, and network security risks, financial management risks have the highest weight, and there is little difference between operational risks and network security risks, which means that financial management risks are particularly important in risk assessment. The most important task is to prevent capital risk and money laundering risk and then cash-out risk.

The corresponding risk weights can be ranked for enterprise risks. Figure 10 displays the ranking results.

Figure 10 signifies that financial management risk and network security risk are high-level risks, weights of which are 0.43 and 0.33 , respectively. Market safety risk is a low risk, accounting for about $0.24 \%$ of the total. The reason why financial risk becomes a high-level risk is related to the current market management because the relevant legal documents have a certain lag. Now, with the continuous development of Internet technology, the trend of cashing out and money laundering is prevalent. Some people want to transact illegal money through these informal techniques, which makes MP have great financial management risks. The reason why network security risks become advanced risks is mainly the immaturity of related technologies, the imperfection of the system, and the constant leakage of information that some criminals have the opportunity to exploit this loophole, and therefore use the leaked information for financial fraud. The middle-aged and elderly people are not very aware of fraud prevention, so these people have become the targets of criminals. This is one of the reasons why the number of middle-aged and elderly people choosing MP is small. The reason why market financial risks become lower risks is that there are relatively few financial risks and accidents under market control, some potential entrants have not yet entered the market, and there are not many big competitors in the industry. If the existing enterprises have

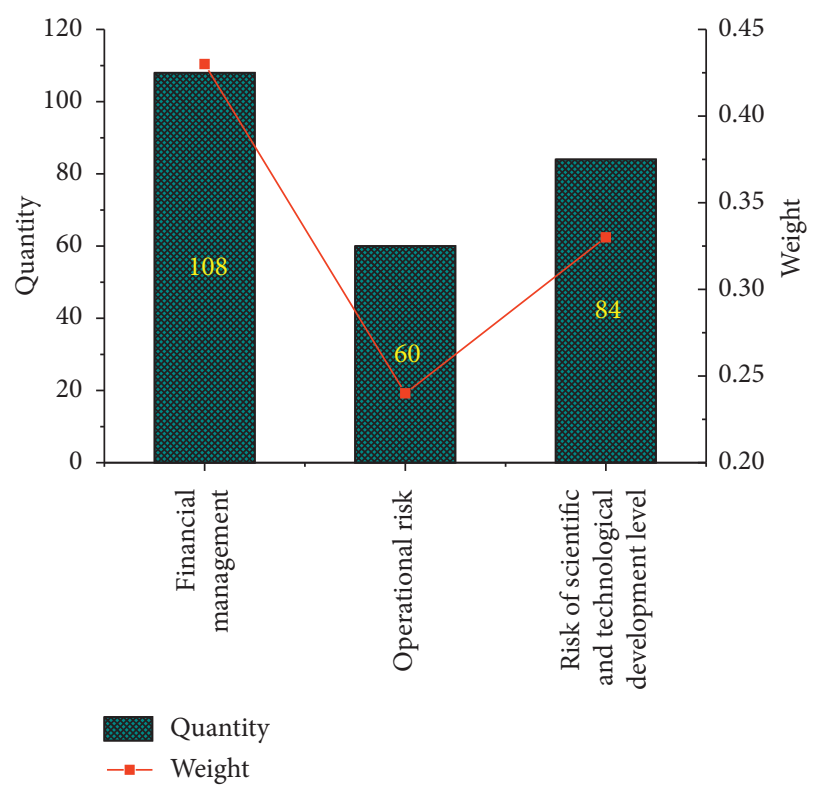

Figure 10: Results of level 1 type evaluation.

great risks, consumers will lose confidence in MP, and it is very possible to choose other payment methods, so it is particularly important to ensure the market security of MP.

Similarly, it is necessary to assess the risk of the secondary type, and Figure 11 shows specific results.

Figure 11 indicates that among the financial management risks, the main one is capital risk, of which the weight is about 0.435 , then money laundering risk weights for about 0.313 of the total proportion, and finally cash-out risk weights for about 0.252 . Among the market security risks, the platform operation risk weights for about $0.405 \%$. Next, credit risk weights for about 0.309 , and finally, reputation risk weights for about 0.286 . Among the network security risks, software and hardware risks account for a relatively large proportion, which is about 0.472 , followed by information security risks weighting for about 0.411 . Finally, business innovation risks weights for about 0.117 . Financial management risk, operational risk, and network security risk are all important factors that affect the rapid development of MP. There are some specific factors that jointly hinder the development of MP, and some risks are uncontrollable and inevitable, so it is necessary to do a good prevention in advance.

To sum up, from the above evaluation results, the value of the maximum weight is 0.43 , which corresponds to the financial management risk and belongs to the advanced risk. Followingly, 0.33 corresponds to the operational risk, which belongs to the advanced risk. In the secondary type, more than half of the total proportion is high risk and higher risk, and about $20 \%$ is medium risk. About $30 \%$ risks belong to low risk. From these data, it can be concluded that at present, China's MP enterprises have higher risks, especially the financial management risks of enterprises. This phenomenon is also consistent with the common enterprise risk events today. Residents, businesses, and the government should jointly guard against the deterioration of this 


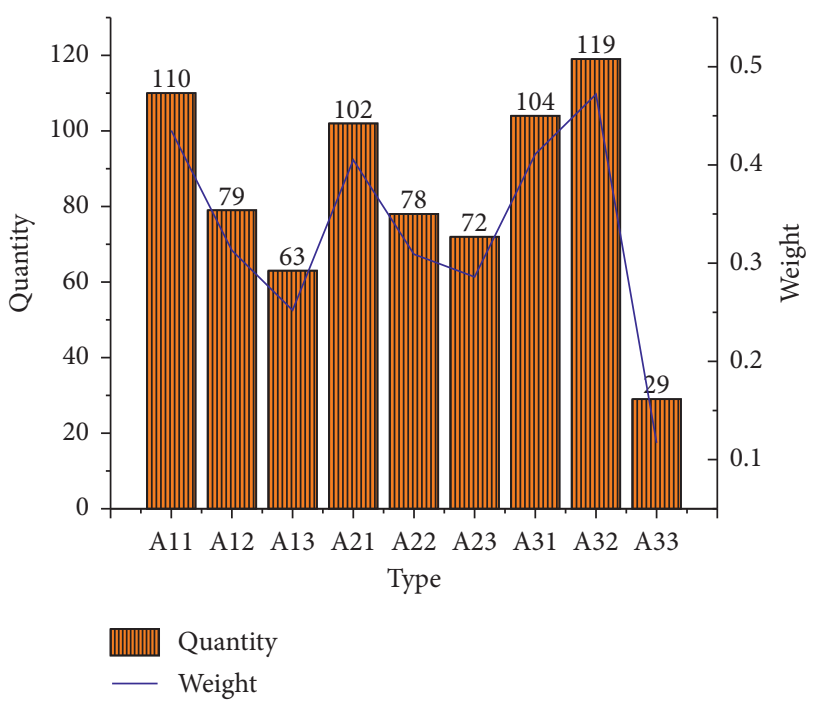

FIGURE 11: Evaluation results of secondary type.

phenomenon. What can be done is to minimize the social risks brought by MP to the maximum extent.

3.3. Suggestions on MP Risk Prevention. (1) As a government, it is necessary to strengthen management and control. Specifically, laws and regulations can help establishing a process plan suitable for risk supervision of MP enterprises. To severely crack down on Internet money laundering and cash-out activities, it is necessary for the government and financial institutions to unite and work out a method that is in line with the development of national conditions and can actually solve problems to provide a healthy MP environment for the society. Tracking of capital circulation and supervision on the abnormal capital circulation should be increased and made in time. Meanwhile, the relevant government units can also set up special investigation teams and employ special technical personnel to investigate the market security of MP. There is also a need to build a sound market access mechanism. Whenever an enterprise enters the MP market, it should be strictly invested on its credit and reputation, and it should be recorded to create vitality for the development of the MP market. (2) As an enterprise, it should actively respond to the national policy and establish a correct concept of MP. Enterprises should resolutely oppose online money laundering and cash-out activities and immediately report to relevant units if they encounter bad behaviors of criminals. Continuous optimization should be made on the construction of risk management system within the enterprise. The division of labor of each department should be clarified, and the process of enterprise financial management should be standardized. Additionally, the company's network security platform should be checked and updated in time. If it is invaded by hackers, a large amount of customer data will be leaked, which is very hazardous to the development of MP. (3) Citizens should know how to defend their rights. When their legitimate rights and interests are infringed, they should immediately take up legal weapons to protect themselves, which not only ensures their rights and interests but also reduces the harm brought by MP to society. Meanwhile, constant learning of the knowledge of safeguarding rights from cyber fraud stops criminals from having an opportunity. Finally, the whole society should try best to let the middle-aged and elderly people use cash payment to prevent MP from being defrauded of their property and so on.

\section{Conclusions}

Mobile payment is a "double-edged sword," which not only brings convenience to human beings but also brings some problems. From the perspective of AI, analyzes and evaluation of the financial leverage are made for enterprises under MB. Conclusions are drawn as follows: (1) the research data are collected by using the questionnaire survey method. From these data, it is found that the main risks to enterprise finance are financial management risk, operation risk, and network security risk. (2) After classifying the relevant risk weights, it is found that the weight of financial management risk is the largest, and the difference between operation risk and network security risk is small. (3) After sorting the risk weights, it is observed that financial management risk and network security risk belong to advanced risk, which are 0.43 and 0.33 , respectively. Market security risk is low risk, accounting for about 0.24 of the totals. Besides, in the financial management risk, the main weight is the capital risk, accounting for about 0.435 of the total proportion. (4) In view of the existing mobile payment risks in enterprises, the corresponding opinions and suggestions are given from the perspective of government, businesses, and residents [35].

Due to limited energy, there are still some shortages in the acquisition of relevant data, resulting in deviations in the test of the results, and there is no discussion on the economic benefits of mobile payment risk assessment. Subsequently, the corresponding assessment can be carried out according to the specific situation, so that the final suggestions and opinions can promote the long-term and stable development of enterprise financial management.

\section{Data Availability}

The data used to support the findings of this study are available from the corresponding author upon request.

\section{Conflicts of Interest}

The authors declare that they have no conflicts of interest.

\section{References}

[1] H. Zhao and I. A. Mensah, "Panel econometric analysis on mobile payment transactions and traditional banks effort toward financial accessibility in sub-sahara africa," Sustainability, vol. 171, no. 4356, pp. 737-738, 2020.

[2] W. Dan, "Battles of mobile payment networks: the impacts of network structures, technology complementarities and institutional mechanisms on consumer loyalty," IEEE Syst J, vol. 12, no. 1, pp. 114-121, 2018. 
[3] Z. Pi, "State of the art: secure mobile payment," IEEE Access, vol. 116, no. 6, Article ID 061102, 2018.

[4] H. Zhou, "Emv-compatible offline mobile payment protocol with mutual authentication," Sensors, vol. 699, no. 1, p. 12049 , 2018.

[5] Z. Kahn, "Why do people switch mobile payment service platforms? an empirical study in Taiwan," Technology in Society, vol. 1, no. 170, Article ID 112675, 2019.

[6] F. A. Azizan, A. M. Kiloes, I. S. Astuti, and A. Abdul Aziz, "Application of optical remote sensing in rubber plantations: a systematic review," Remote Sensing, vol. 13, no. 3, p. 429, 2021.

[7] B. B. Gupta and S. Narayan, "A key-based mutual authentication framework for mobile contactless payment system using authentication server," Journal of Organizational and End User Computing, vol. 33, no. 2, pp. 1-16, 2021.

[8] S.-H. Liao and C.-H. Ho, "Mobile payment and mobile application (app) behavior for online recommendations," Journal of Organizational and End User Computing, vol. 33, no. 6, pp. 1-26, 2021.

[9] X. Jiang, "Management and optimization of enterprise financial risk under the background of big data 2020 international conference on social sciences and big data application," IEEE Access, vol. 116, no. 6, Article ID 061102, 2018.

[10] W. Thomas, "Enterprise financial risk management platform based on $5 \mathrm{G}$ mobile communication and embedded system," Microprocessors and Microsystems, vol. 13, no. 3, p. 429, 2019.

[11] W. Cao, "Analysis on the problems and countermeasures of enterprise financial risk management in China:taking zhejiang Xiang piaopiao food Co. Ltd. As an example," Journal of Lianyungang Technical College, vol. 699, no. 1, p. 12049, 2020.

[12] J. Hao, "The history of enterprise risk management at hydro one inc," Journal of Risk and Financial Management, vol. 14, no. 8, pp. 370-373, 2019.

[13] S. Chen, "Effect of financial risk management on the performance of insurance companies in Rwanda (2015-2019) SSRN electronic journal," Telecommunications Policy, vol. 30, no. 420 , pp. 88-96, 2019.

[14] L. Nelloh, "Will users keep using mobile payment? it depends on trust and cognitive perspectives," Procedia Computer Science, vol. 14, no. 2, pp. 124-128, 2019.

[15] M. Hsiao, "Mobile payment services as a facilitator of value co-creation: a conceptual framework. Journal of High Technology Management Research," Science Observation, vol. 15, no. 06, pp. 79-80, 2020.

[16] L. Liao, "Mobile payment and online to offline retail business models. Journal of Retailing and Consumer Services," Popular Science and Technology, vol. 22, no. 254, pp. 6-8, 2020.

[17] X. Gong, "Transition from web to mobile payment services: the triple effects of status quo inertia," International Journal of Information Management," vol. 25, no. 1, pp. 102-112, 2020.

[18] Y. Wang, "Mobile payment and informal business: evidence from China's household panel data," Progress in Laser and Optoelectronics, vol. 57, no. 663, pp. 102-108, 2020.

[19] O. Vincent, "An identity-based elliptic curve cryptography for mobile payment security," Computer Science, vol. 39, no. 208, pp. 112-119, 2020.

[20] S.-B. Tsai, Y. Xue, J. Zhang et al., "Models for forecasting growth trends in renewable energy," Renewable and Sustainable Energy Reviews, vol. 77, pp. 1169-1178, 2017.

[21] D. Jia, "Influence of habits on mobile payment acceptance: an ecosystem perspective," Information Systems Frontiers, vol. 26, no. 1, pp. 200-207, 2018.
[22] Y.-H. Yuan, S.-H. Tsao, J.-T. Chyou, and S.-B. Tsai, "An empirical study on effects of electronic word-of-mouth and Internet risk avoidance on purchase intention: from the perspective of big data," Soft Computing, vol. 24, no. 8, pp. 5713-5728, 2020.

[23] B. Esfahani, "The influence of individual differences on nfcbased mobile payment adoption in restaurant industry," IEEE Journal of Selected Topics in Applied Earth Observations and Remote Sensing, vol. 1, no. 99, pp. 1-2, 2021.

[24] S. Verma, "The effect of government regulations on continuance intention of in-store proximity mobile payment services," "International Journal Of Bank Marketing, vol. 25, no. 1, pp. 102-112, 2020.

[25] W. Nelloh, "Will users keep using mobile payment? it depends on trust and cognitive perspectives," Procedia Computer Science, vol. 699, no. 1, p. 12049, 2021.

[26] N. Bojjagani, "A secure end-to-end proximity nfc-based mobile payment protocol," Xiehe Medical Journal, vol. 12, no. 1, pp. 114-121, 2021.

[27] K. Chalee, "A lightweight and secure nfc-base mobile payment protocol ensuring fair exchange based on a hybrid encryption algorithm with formal verification. International Journal of Communication Systems," Economic Management Digest, vol. 754, no. 16, pp. 161-162, 2020.

[28] M. Zhang, "Spillover effects from web to mobile payment services: the role of relevant schema and schematic fit," Internet Research, vol. 21, no. 8, p. 2695, 2021.

[29] I. Khn, "Mobile payment - eine vertrauensfrage," Textil Wirtschaft, vol. 699, no. 1, p. 12049, 2021.

[30] M. Deng, "The specific application of microelectronic control electromechanical equipment in industry," Electronics World, vol. 08 , no. 590 , pp. $152-153,2020$.

[31] C. Wang, J. H. Fan, and S. M. Lin, "Research on the correlation between optical remote sensing vegetation index and sar remote sensing parameters and its main influencing factors," Remote sensing of land and resources, vol. 32, no. 126, pp. 134-141, 2020.

[32] A. Mk, "Technological factors of mobile payment: a systematic literature review," Procedia Computer Science, vol. 13, no. 3, p. 429, 2021.

[33] N. Sinha, "Assessment of mobile technology use in the emerging market: analyzing intention to use m-payment services in India," Telecommunications Policy, vol. 28, no. 424, pp. 88-91, 2020.

[34] S. Bojjagani, "Systematic survey of mobile payments, protocols, and security infrastructure," Journal of Ambient Intelligence and Humanized Computing, vol. 39, no. 289, pp. 242-246, 2020.

[35] A. Guo, "Proposition of a shared and value-oriented work structure for hospital-based health technology assessment and enterprise risk management processes," International Journal of Technology Assessment in Health Care, vol. 699, no. 1, p. 149, 2018. 